\title{
Diagnostic Value of Magnetic Resonance Imaging in Prediction of Malignancy in Adnexal Masses
}

\author{
Zarife Kerimova, Sunullah Soysal ${ }^{*}$ \\ Marmara University Department of Obstetrics and Gynecology
}

\begin{abstract}
Ovarian cancer is the leading cause of death in women among gynecological cancers. For this reason adnexal masses diagnosis is important to make differentiation of malignant lesions from benign lesions. Ultrasound is generally used as a second step of evaluation of adnexal masses after pelvic examination. Unfortunately ultrasound may not be enough to detect malignant lesions in adnexa. For this reason several diagnostic approaches are being used. Magnetic resonance imaging can be used in adnexal masses which have malignancy suspicion. This study aims to show the diagnostic value of MRI in diagnosis of adnexal masses.

Files of 98 patients who underwent surgery for adnexal masses were retrospectively investigated. Patients under 18 years of age, patients whose adnexal mass were incidentally diagnosed during surgery and patients who don't have preoperative imaging studies were excluded from the study. Postoperative pathology results and preoperative MRI findings were compared.

For MRI evaluation of the patients; the contrast enhancement was found to be more positive in masses diagnosed as malignant (44\%vs.6.4\%). Also presence of both omental cake and free fluid was found to be related with diagnosis of malignancy in final result. Bilaterally located masses in MRI were found to be more malignant (57\%vs.27.14\%).

When MRI finding were evaluated as malignant or benign the consistency with the final pathology results were studied. A moderate correlation with the final results was found with the MRI results. MRI can be used in differentiation of malignant adnexal masses.
\end{abstract}

Key Words: Adnexal mass, MRI, Malignancy

\section{Introduction}

Part of the ovary or an adnexal mass that is detected during ultrasound which is inconsistent with normal physiology can be defined as adnexal lesion (1). Adnexal masses may present themselves with many symptoms. These lesions are important in respect to malignant potential. Among gynecological cancers ovarian cancers are the leading cause of mortality. Every one woman in 95 has the risk of ovarian cancer in life time. Unfortunately majority of the patients with ovarian cancer are diagnosed in stage III or IV and 5 years survival rate decreases from $90 \%$ (in women cancer confined to ovary Stage I) to 30$73 \%$ by this stage shift (2). For this reason malignant potential of the adnexal masses are very important. Adnexal masses can be incidentally diagnosed; a woman has a life time risk of undergoing surgery for an incidentally diagnosed adnexal mass is 5-10 \%. The incidentally diagnosed adnexal masses are challenging because of difficulty in differentiating whether it is malignant or benign $(3,4)$.
Ultrasound is generally used for detection of suspicious adnexal lesions during physical examination or adnexal lesions are diagnosed during an ultrasound performed for another reason. In diagnosis of malignancy MRI has a sensitivity and specificity of $100 \%$ and $94 \%$ respectively. MRI provides a confident diagnosis of adnexal lesions in benign nature $(5,6)$.

The aim of this study is to evaluate diagnostic value of MRI in adnexal masses.

\section{Materials and Methods}

This study was conducted in a tertiary center in between 2011 and 2017. Patients with adnexal mass/es were included in the study. Files of 98 patients who underwent surgery for adnexal masses were retrospectively investigated. Patients under 18 years of age, patients whose adnexal mass were incidentally diagnosed during surgery and patients who don't have preoperative imaging studies were excluded from the study. Demographic features of the patients, ultrasound findings, MRI findings, intraoperative frozen 
Table 1. Demographic features of patients

\begin{tabular}{|c|c|c|c|c|}
\hline & & Median & Min & Max \\
\hline Age & & 45 & 18 & 80 \\
\hline Gravida & & 3 & 0 & 15 \\
\hline Parity & & 2 & 0 & 9 \\
\hline Abort & & 0 & 0 & 9 \\
\hline \multirow{2}{*}{ Menopausea } & Pre-menopausal & 56 & \multicolumn{2}{|c|}{57.7} \\
\hline & Post-menopausal & 41 & \multicolumn{2}{|c|}{42.3} \\
\hline \multirow{3}{*}{ Complainta } & No complaint & 25 & \multicolumn{2}{|c|}{25.8} \\
\hline & Abdomino-pelvic pain & 69 & \multicolumn{2}{|c|}{71.1} \\
\hline & Vaginal bleeding & 3 & \multicolumn{2}{|c|}{3.1} \\
\hline
\end{tabular}

Table 2. Ultrasound features of the adnexal masses

\begin{tabular}{lccc}
\hline & & $\mathrm{n}$ & $\%$ \\
\hline \multirow{3}{*}{ Location of the mass } & Right & 41 & 42.3 \\
& Left & 43 & 44.3 \\
Free Fluid & Bilateral & 13 & 13.4 \\
& Present & 19 & 19.6 \\
& Absent & 78 & 80.4 \\
Feature of the Mass & Pure Cystic & 6 & 6.2 \\
& Solid & 30 & 30.9 \\
& Mix & 15 & 15.5 \\
& Septated & 10 & 10.3 \\
Extra finding & Heterogenic & 36 & 37.1 \\
& Uterine mass & 7 & 7.2 \\
Size 1a & Mass in other organ & 3 & 3.1 \\
Size 2 a & No Extra finding & 86 & 88.7 \\
Mean Size a & Intraabdominal mass & 1 & 1.0 \\
\hline
\end{tabular}

${ }^{a}$ for continuous data $\%$ is used instead of minimum and maximum values and median is used for $\mathrm{n}$

section results and final pathology results were recorded. Ethical approval was taken from the universities local ethics committee.

Ultrasound findings were recorded as the side of the mass, presence of the free fluid in the abdominal cavity, nature of the mass (cystic/solid) and the two dimensional sizes. MRI findings were the contrast enhancement of the lesion, side of the lesion, presence of the additional findings.

Statistical Analysis: SPSS version 23.0 was used for analysis of the data. Normal distribution of the variables was evaluated with visual (histogram and probability graphics) and analytic methods (Kolmogorov-Smirnov/Shapiro-Wilk tests). Descriptive analysis was given by usage of mean and standard deviation for normally distributed variables. Pearson Chi-square and Fisher's Exact tests were used. If the normal distribution of the variables are not shown Mann Whitney $U$ test was used. ROC analysis was used to define diagnostic cut off levels for MRI. Conditions in which p level is less than 0.05 , is accepted as statistically significant.

\section{Results}

Ninety seven patients with adnexal mass or masses were included in the study. Median age for all patients was 45 (18-80). Fifty six patients were in premenopausal state and 41 of them were in postmenopausal state. Seventy one percent of the patients had abdominopelvic pain and 25\% patient had no complaints. (Table 1) shows the demographic 
Table 3. Intraoperative and postoperative pathological results of adnexal masses

\begin{tabular}{lccc}
\hline & & $\mathrm{n}$ & $\%$ \\
\hline Intraoperative Frozen Result & Malignant & 23 & 23.7 \\
& Benign & 66 & 68.0 \\
& Left for paraffin & 2 & 2.1 \\
Final Result & Borderline & 6 & 6.2 \\
& Malignant & 25 & 25.8 \\
& Benign & 72 & 74.2 \\
& Borderline & 0 & 0.0 \\
\hline
\end{tabular}

Table 4. MRI features of adnexal masses

\begin{tabular}{|c|c|c|c|c|}
\hline & & $\mathrm{n}$ & & \\
\hline \multirow{2}{*}{$\begin{array}{l}\text { Contrast } \\
\text { enhancement }\end{array}$} & Present & 50 & \multicolumn{2}{|c|}{51.5} \\
\hline & Absent & 47 & \multicolumn{2}{|c|}{48.5} \\
\hline \multirow{4}{*}{ Extra findings } & Omental Cake & 1 & \multicolumn{2}{|c|}{1.0} \\
\hline & Free fluid & 10 & \multicolumn{2}{|c|}{10.3} \\
\hline & Fluid + Omental Cake & 13 & \multicolumn{2}{|c|}{13.4} \\
\hline & No Extra finding & 73 & \multicolumn{2}{|c|}{75.3} \\
\hline \multirow{3}{*}{ MRI location } & Right & 36 & \multicolumn{2}{|c|}{37.1} \\
\hline & Left & 47 & \multicolumn{2}{|c|}{48.5} \\
\hline & Bilateral & 14 & \multicolumn{2}{|c|}{14.4} \\
\hline MRI size-1 a & & 76.0 & 31.0 & 200.0 \\
\hline MRI size-2 a & & 65.0 & 25.0 & 170.0 \\
\hline MRI mean size a & & 68.5 & 30.5 & 180.0 \\
\hline
\end{tabular}

${ }^{a}$ for continuous data $\%$ is used instead of minimum and maximum values and median is used for $\mathrm{n}$

findings and the complaints of the patients.

Ultrasound findings of the patients were evaluated. Forty two percent of the lesions were located in right side and $44 \%$ of the lesions were located in the left side and $13 \%$ of the lesions were located on both sides. In $80 \%$ of the cases there was no free fluid in the abdominal cavity. Six percent of the cases was pure cystic, $30 \%$ was solid, $15 \%$ was mixed type and $37 \%$ defined as heterogeneous in nature. Seven patients had an accompanying uterine mass and 3 of them had accompanying lesion in other organs. Median sizes for masses were $73 \mathrm{~mm}, 67 \mathrm{~mm}$ for two dimensions and the median of two dimensions was 67 $\mathrm{mm}$. (Table 2) shows the ultrasound features of the adnexal masses.

Pathological findings of the masses are divided into two. The intraoperative frozen section results were; $23 \%$ were malignant and 66 were benign in nature. Six patients were diagnosed as borderline tumors and 2 patients diagnosis were left for final diagnosis after paraffin sections. The final pathology results were; $25 \%$ were malignant, $74 \%$ were benign in nature and none of the patients had the diagnosis of borderline tumor in final pathology results. (Table 3) shows the malignancy patterns of the adnexal masses intraoperatively and postoperatively.

When MRI findings were evaluated $51 \%$ of the masses had contrast enhancement, omental cake was found only in one patient and 10 patients had free fluid in abdominal cavity and 13 patients had both omental cake appearance and free fluid in the abdominal cavity. Forty eight $\%$ of the patients had masses located in the left side and 14\% had bilateral lesions. (Table 4) shows the MRI features of adnexal masses.

When the final pathology results in respect to preoperative imaging findings and demographical features of the patients were evaluated, the increased age was shown to increase malignancy rate. Thirty six percent of the masses detected during 
Kerimova and Soysal/ Diagnosis of adnexal malignancies with Magnetic Resonance Imaging

Table 5. Evaluation of measurable variables according to pathology result

\begin{tabular}{|c|c|c|c|c|c|c|c|}
\hline & \multicolumn{6}{|c|}{ Pathology result } & \multirow{3}{*}{$\mathrm{p}$} \\
\hline & \multicolumn{3}{|c|}{ Malignant } & \multicolumn{3}{|c|}{ Benign } & \\
\hline & Median & Min & Max & Median & Min & Max & \\
\hline Age & 50 & 23 & 80 & 44 & 18 & 73 & 0.042 \\
\hline Gravida & 3 & 0 & 15 & 3 & 0 & 12 & 0.320 \\
\hline Parity & 3 & 0 & 9 & 2 & 0 & 9 & 0.305 \\
\hline Abort & 0 & 0 & 6 & 0 & 0 & 9 & 0.408 \\
\hline USG size -1 & 90.0 & 30.0 & 250.0 & 70.0 & 31.0 & 214.0 & 0.509 \\
\hline USG size -2 & 70.0 & 30.0 & 200.0 & 65.5 & 24.0 & 180.0 & 0.304 \\
\hline USG mean size & 81.5 & 35.0 & 225.0 & 66.5 & 28.0 & 190.0 & 0.428 \\
\hline MRI size -1 & 73.0 & 39.0 & 200.0 & 77.0 & 31.0 & 200.0 & 0.849 \\
\hline MRI size -2 & 72.0 & 42.0 & 140.0 & 65.0 & 25.0 & 170.0 & 0.283 \\
\hline MRI mean size & 78.5 & 45.5 & 170.0 & 67.7 & 30.5 & 180.0 & 0.520 \\
\hline
\end{tabular}

Mann-Whitney U test

postmenopausal period was found to be malignant, only $18 \%$ of the masses detected during premenopausal period was found to be malignant $(p<0.05)$. Among patients who has free fluid in the ultrasound, $78 \%$ was found to be malignant and only $12 \%$ of patients who did not have free fluid in the ultrasound was found to be malignant $(\mathrm{p}<0.05)$. Solid lesions under ultrasound view were found to be more malignant than the pure cystic lesions $(\mathrm{p}<0.05)$. All patients $(\mathrm{n}=3)$ who have extra finding in other organs were noted during ultrasound were found to be malignant in final pathology results $(\mathrm{p}<0.05)$.

For MRI evaluation of the patients; the contrast enhancement was found to be more positive in masses diagnosed as malignant (44\%vs.6.4\%) $(p<0.05)$. Also presence of both omental cake and free fluid was found to be related with diagnosis of malignancy in final result $(p<0.05)$. Bilaterally located masses in MRI were found to be more malignant $(57 \%$ vs.27,14\%) $(\mathrm{p}<0.05)$. (Table 5) and (Table 6) shows evaluation of variables according to pathology result.

When MRI finding were evaluated as malignant or benign the consistency with the final pathology results were studied. A moderate correlation with the final results was found with the MRI results (Kappa score:0.715) $(\mathrm{p}<0.05)$.

\section{Discussion}

Ovaries are pelvic organs that are hard to be reached during pelvic examination. Bimanual pelvic examination is not enough for detection of adnexal masses in early stages. The pelvic examination may be insufficient especially for lesions less than $5 \mathrm{~cm}$. Those lesions can be detected with ultrasound (7). Currently together with ultrasound new markers increased the diagnostic accuracy of adnexal masses.

Grab et al. (8) evaluated the diagnostic value of the ultrasound, MRI and positron emission tomography (PET) in adnexal masses. They reported that addition of MRI and PET increased the diagnostic accuracy of the adnexal masses. We also evaluated the diagnostic value of the MRI in adnexal masses in respect to malignancy.

Median age in the present study was found as 45 and $42 \%$ was in postmenopausal state. Similarly Meray et al (9) found median age as 43,5. Ashley et al, (10) revealed a median age of 46 in a similar study population. MRI is preferred as a second step diagnostic modality in differentiation of the complex adnexal masses. In several studies MRI was found to be superior to ultrasound in respect to malignancy detection (11-13). We found sensitivity and specificity of MRI 85 and $88 \%$ respectively. The positive predictive value was detected as $77.2 \%$ and negative predictive value was detected as 92.6 for MRI in diagnosis of adnexal masses. Grab et al found sensitivity $83 \%$ and specificity $84 \%$ with a PPV $42 \%$ and NPV as 97\% [8]. Komatsu et al (12) found sensitivity as $100 \%$ and specificity as $98 \%$. Russel et al reported sensitivity of $90 \%$ and specificity of $88 \%$ with a PPV of $98 \%$ and NPV of $23 \%$ (14). Trappen et al also showed a sensitivity of $78 \%$ and specificity of $86 \%$ for diagnosis adnexal masses (10). Nam et al also found a sensitivity of $82.5 \%$ and specificity of

East J Med Volume:24, Number:1, January-March/2019 
Table 6. Evaluation of non-measurable variables according to pathology results

\begin{tabular}{|c|c|c|c|c|c|c|}
\hline & & \multicolumn{4}{|c|}{ Pathology result } & \multirow{3}{*}{ p } \\
\hline & & \multicolumn{2}{|c|}{ Malignant } & \multicolumn{2}{|c|}{ Benign } & \\
\hline & & $\mathrm{n}$ & $\%$ & $\mathrm{n}$ & $\%$ & \\
\hline \multirow{2}{*}{ Menopausal state } & Premenopausal & 10 & 17.9 & 46 & 82.1 & \multirow{2}{*}{$0.037 \mathrm{a}$} \\
\hline & Postmenopausal & 15 & 36.6 & 26 & 63.4 & \\
\hline \multirow{3}{*}{ Complaint } & No complaint & 6 & 24 & 19 & 76 & \multirow{3}{*}{$0.743 \mathrm{~b}$} \\
\hline & Abdominopelvic pain & 19 & 27.5 & 50 & 72.5 & \\
\hline & Bleeding & 0 & 0 & 3 & 100 & \\
\hline \multirow{3}{*}{ Mass location in ultrasound } & Right & 10 & 24.4 & 31 & 75.6 & \multirow{3}{*}{$0.005 \mathrm{a}$} \\
\hline & Left & 7 & 16.3 & 36 & 83.7 & \\
\hline & Bilateral & 8 & 61.5 & 5 & 38.5 & \\
\hline \multirow{2}{*}{ Free fluid in ultrasound } & Present & 15 & 78.9 & 4 & 21.1 & \multirow{2}{*}{$<0.001 \mathrm{~b}$} \\
\hline & Absent & 10 & 12.8 & 68 & 87.2 & \\
\hline \multirow{2}{*}{\multicolumn{2}{|c|}{$\begin{array}{l}\text { Pure cystic } \\
\text { Solid }\end{array}$}} & 0 & 0 & 6 & 100 & \multirow{5}{*}{$0.008 \mathrm{~b}$} \\
\hline & & 14 & 46.7 & 16 & 53.3 & \\
\hline Feature of the Mass in ultrasour & Mix & 5 & 33.3 & 10 & 66.7 & \\
\hline & Septated & 2 & 20 & 8 & 80 & \\
\hline & Heterogenic & 4 & 11.1 & 32 & 88.9 & \\
\hline \multirow{4}{*}{ Extra finding in ultrasound } & Uterine mass & 1 & 14.3 & 6 & 85.7 & \multirow{4}{*}{$0.029 \mathrm{~b}$} \\
\hline & Mass in other organ & 3 & 100 & 0 & 0 & \\
\hline & No Extra finding & 21 & 24.4 & 65 & 75.6 & \\
\hline & Intraabdominal mass & 0 & 0 & 1 & 100 & \\
\hline \multirow{2}{*}{ Contrast enhancement in MRI } & Present & 22 & 44 & 28 & 56 & \multirow{2}{*}{$<0.001 \mathrm{a}$} \\
\hline & Absent & 3 & 6.4 & 44 & 93.6 & \\
\hline \multirow{4}{*}{ Extra findings in MRI } & Omental Cake & 1 & 100 & 0 & 0 & \multirow{4}{*}{$<0.001 \mathrm{~b}$} \\
\hline & Free fluid & 4 & 40 & 6 & 60 & \\
\hline & Fluid+ Omental Cake & 9 & 69.2 & 4 & 30.8 & \\
\hline & No Extra finding & 11 & 15.1 & 62 & 84.9 & \\
\hline \multirow{3}{*}{ Location of mass in MRI } & Right & 10 & 27.8 & 26 & 72.2 & \multirow{3}{*}{$0.006 \mathrm{a}$} \\
\hline & Sol & 7 & 14.9 & 40 & 85.1 & \\
\hline & Bilateral & 8 & 57.1 & 6 & 42.9 & \\
\hline
\end{tabular}

a Chi-square test, b Fisher's exact test

Table 7. Sensitivity and specificity of MRI in diagnosis of adnexal masses in different studies

\begin{tabular}{|c|c|c|c|c|c|c|}
\hline Study & Year & $\mathrm{N}$ & Sensitivity & Specificity & PPV & NPV \\
\hline Komatsu et al. & 1990 & 82 & 100 & 98 & - & - \\
\hline Grab et al. & 2000 & 101 & 83 & 84 & 42 & 97 \\
\hline Russell et al. & 2005 & 76 & 90 & 88 & 98 & 23 \\
\hline Trappen et al. & 2007 & 196 & 78 & 86 & - & - \\
\hline Nam et al. & 2010 & 95 & 82.5 & 63.6 & 86.7 & 56 \\
\hline Haggerty et al. & 2014 & 237 & 95 & 94.1 & - & - \\
\hline Kerimova et al. & 2017 & 97 & 85 & 88.3 & 77.2 & 92.6 \\
\hline
\end{tabular}


63.8 with a PPV of $86.7 \%$ and NPV of $56 \%$ (15). Haggerty et al studied largest population number among all studies that were discussed, they found 95\% sensitivity and $94.1 \%$ specificity for diagnosis of adnexal masses with usage of MRI. (Table 7) shows different studies showing sensitivity and specificity of MRI in diagnosis of adnexal masses.

The pathological results are moderately correlated with MRI findings in our study. We prefer performing a MRI study before surgery to refer patient to a gynecologic oncology expert.

\section{References}

1. Liu JH, Zanotti KM. Management of the adnexal mass. Obstetrics \& Gynecology 2011; 117: 1413-1428.

2. Horner ML, Ries M, Krapcho N, et al., SEER Cancer Statistics Review, 1975-2006, National Cancer Institute. Bethesda MD. 2009.

3. McDonald JM, Modesitt SC. The incidental postmenopausal adnexal mass. Clin Obstet Gynecol 2006; 49: 506-516.

4. Jeong YY, Outwater EK, Kang H.K. Imaging evaluation of ovarian masses. Radiographics 2000; 20: 1445-1470.

5. Adusumilli S, Hussain HK, Caoili EM, et al. MRI of sonographically indeterminate adnexal masses. American Journal of Roentgenology 2006; 187: 732-740.

6. Kinkel K, Lu Y, Mehdizade A, Pelte MF, Hricak H. Indeterminate ovarian mass at US: incremental value of second imaging test for characterization-meta-analysis and Bayesian analysis. Radiology 2005; 236: 85-94.

7. Murta E, Da Silva C, R. Gomes B. TavaresMurta, Melo A. Ultrasonographic criteria and tumor marker assay are good procedures for the diagnosis of ovarian neoplasia in preselected outpatients. European journal of gynaecological oncology 2003; 25: 707-712.
8. Grab D, Flock F, Stöhr I, et al.. Classification of asymptomatic adnexal masses by ultrasound, magnetic resonance imaging, and positron emission tomography. Gynecologic oncology 2000; 77: 454-459.

9. Meray O. Adneksiyal kitlelerin malign-benign ayrımında malignite risk indeksi (RMI)'nin prediktif değeri, in Kadin Hastalıkları ve Doğum Anabilim Dalı. 2009, İnönü Üniversitesi: Malatya.

10. Haggerty AF, Hagemann AR, Chu C, Siegelman ES, Rubin SC. Correlation of pelvic magnetic resonance imaging diagnosis with pathology for indeterminate adnexal masses. International Journal of gynecological cancer 2014; 24: 1215-1221.

11. Yamashita Y, Torashima M, Hatanaka Y. et al. Adnexal masses: accuracy of characterization with transvaginal US and precontrast and postcontrast MR imaging. Radiology 1995; 194: 557-565.

12. Komatsu T, Konishi I, Mandai M, et al. Adnexal masses: transvaginal US and gadolinium-enhanced MR imaging assessment of intratumoral structure. Radiology 1996; 198: 109-115.

13. Hata K, Hata T, Manabe A, Sugimura K, Kitao M. A critical evaluation of transvaginal Doppler studies, transvaginal sonography, magnetic resonance imaging, and CA 125 in detecting ovarian cancer. Obstetrics \& Gynecology 1992; 80: 922-926.

14. Low RN, Duggan B, Barone R.M, Saleh F, Song ST. Treated ovarian cancer: MR imaging, laparotomy reassessment, and serum CA-125 values compared with clinical outcome at 1 year. Radiology 2005; 235: 918-926.

15. Nam EJ, Yun MJ, Oh YT, et al. Diagnosis and staging of primary ovarian cancer: correlation between PET/CT, Doppler US, and CT or MRI. Gynecologic oncology 2010; 116: 389394. 